\title{
A New Method for the Determination of Creatinine in Urine Samples Based on Disposable Pipette Extraction
}

\author{
Aline R. Fernandes, Paulo S. de Souza, Anselmo E. de Oliveira and Andréa R. Chaves* \\ Instituto de Química, Universidade Federal de Goiás, 74605-220 Goiânia-GO, Brazil
}

\begin{abstract}
A method based on disposable pipette extraction (DPX) was successfully applied to creatinine determination in urine samples analysis using liquid chromatography with ultraviolet spectrophotometric detection (DPX/LC-UV). DPX variables, number of draw/eject cycles, sample $\mathrm{pH}$, and type of the desorption solvent, were employed in a factorial experimental design to optimize the sorption equilibrium and time analysis. Among the evaluated DPX variables, the highest extraction efficiency was obtained with $500 \mu \mathrm{L}$ of urine sample mixed with $1 \mathrm{~mL}$ of borate solution ( $\mathrm{pH}$ 9) with one draw/eject cycle followed by liquid desorption of $1 \mathrm{~mL}$ of methanol in seven draw/eject cycles. The developed DPX/LC-UV method showed a linear response from the limit of quantification of 0.317 to $3.390 \mathrm{~g} \mathrm{~L}^{-1}$ with $\mathrm{r}^{2}=0.996$ and inter-day precision with a coefficient of variation below $8.8 \%$. Based on these results, the proposed method can be a useful tool for determining the creatinine levels in urine samples.
\end{abstract}

Keywords: creatinine, disposable pipette extraction, urine sample, liquid chromatography

\section{Introduction}

Creatinine (2-amino-1-methyl-2-imidazoline-4-one) is one of the human blood components that is the final product of creatine metabolism in mammals, which is carried out by skeletal muscles to release energy. ${ }^{1}$ Creatinine is extracted from the body by renal excretion at a relatively constant rate. ${ }^{2}$ The variation in creatinine level in blood and urine is an important parameter in clinical diagnostics. Kidney problems, thyroid malfunction, and muscular disorders increase the creatinine concentration in blood serum, and therefore measuring the creatinine concentration in blood, serum, and urine allows diagnosis of these disorders. ${ }^{3}$ Recently, there has been a leap forward towards manufacturing simple, accurate, and reliable biosensors to measure the amount of creatinine. ${ }^{1-3}$ Normal creatinine levels in urine are in the range of 0.407 to $3.054 \mathrm{~g} \mathrm{~L}^{-1}$ in men and 0.373 to $2.545 \mathrm{~g} \mathrm{~L}^{-1}$ in women; however, they can vary according to age and gender. ${ }^{2,4}$ In kidney malfunction, creatinine concentration can exceed $113.118 \mathrm{~g} \mathrm{~L}^{-1}$. Values above $15.836 \mathrm{~g} \mathrm{~L}^{-1}$ require more clinical assays and values above $59.952 \mathrm{~g} \mathrm{~L}^{-1}$ are related to kidney disease. ${ }^{2}$ Patients suffering from kidney disease need to control the creatinine concentration in their blood daily. ${ }^{5}$

Methods to detect concentrations of creatinine have

*e-mail: andrea_chaves@ufg.br been known since at least as early as 1886, when Max Jaffe developed a colorimetric methodology to quantify creatinine within urine. ${ }^{2}$ This is achieved by using potassium bichromate as an indicator, which acts as a colorimeter to elucidate the creatinine concentrations against a set of known standards. ${ }^{2}$ The major limitation of the Jaffe reaction could be attributed to their non-specificity, and as a result, the data are largely inaccurate; biological compounds such as glucose and ascorbic acid can readily interfere with the results. ${ }^{6}$

Lloyd's reagent, that is, aluminum magnesium silicate clay, has been used to selectively adsorb creatinine; nevertheless, substances such as pyruvate and indole produce the interferences in this case. ${ }^{7,8}$ To enhance the specificity, enzymatic methods are often used. ${ }^{9-12}$ The most popular among them is the three-enzyme method, in which creatinine amidohydrolase (CA), creatine amidinohydrolase (CI), and sarcosine oxidase (SO) can be employed to catalyze the hydrolysis of creatinine to hydrogen peroxide $\left(\mathrm{H}_{2} \mathrm{O}_{2}\right)$, which can then be detected amperometrically. ${ }^{9}$ Besides, creatinine iminohydrolase $(\mathrm{CIH})$ was utilized to catalyze the hydrolysis of creatinine to ammonia $\left(\mathrm{NH}_{3}\right)$; the $\mathrm{NH}_{3}$ was then detected by potentiometry. ${ }^{10}$ Although enzymatic methods are much more specific, they are usually expensive and suffer from instability.

Separation methods such as liquid chromatography (LC), gas chromatography, capillary electrophoresis, and 
biosensors based on immobilized enzymes or molecularly imprinted polymers could be alternative methods to creatinine determination with adequate sensibility and specificity. ${ }^{13,14}$ In LC, reversed-phase (RP) and ion-pair chromatography have been the primary separation methods for creatinine in biological fluids. ${ }^{15-17}$

The development of highly efficient analytical instrumentation for the endpoint determination of creatinine from urine samples involves sample pretreatment. This is because most analytical instruments cannot handle sample matrices directly.

The sample preparation technique known as disposable pipette extraction (DPX) is a miniaturized solid-phase extraction (SPE) based device in which a small amount of SPE sorbent is placed inside a pipette tip fitted with a screen at the narrow bottom end and a barrier near the top of the tip. ${ }^{18,19}$ DPX has become an essential tool for the purification and concentration of proteins and peptides in genomics, proteomics, and metabolomics. ${ }^{18-22}$ This technique has also been successfully employed in environmental, toxicological, and drug analyses..$^{18-20,23}$ The DPX extraction efficiency is based on the sorption equilibrium time between the sample solutions and the dispersive sorbent. The choice of washing solvent is based on the type/chemical nature of the sorbent, on the nature of the analyte of interest and in the interferers possibly present in the matrix. Solvent washing is also accomplished by the inflow of air into the nozzle tip. After this stage, finally the elution solvent is sucked into the tip of the nozzle, followed by aspiration of air, several times, in order to ensure the complete desorption of the adsorbed analytes. Consequently, unlike the SPE process, it is not dependent on the sample flow rate. Furthermore, the miniaturized format results in reductions in solvent use and time required compared to the conventional SPE technique. ${ }^{18}$ The main advantage of DPX is its adaptability to high-throughput parallel sample processing while still maintaining flexibility of sorbents and procedures..$^{22}$ In contrast to dispersive-SPE in a tube, which is employed for chemical filtration clean-up (retention of matrix interferences on the sorbent), the DPX format can also be used for analyte retention and elution by a different solvent. The DPX device requires a shorter extraction time, involves less sample manipulation, and provides high recovery and efficiency. The whole process can be automated, including sample injection into the chromatographic system..$^{22}$

The aim of this study was to evaluate DPX microextraction technique, followed by liquid chromatography and ultraviolet spectrophotometric analysis (LC-UV), for the determination of creatinine in urine samples.

\section{Experimental}

Reagents, standards, and samples

Creatinine (99.8\%), uric acid (99.8\%), and sodium acetate $(98.0 \%)$ were obtained from Sigma-Aldrich (St. Louis, Missouri, USA), while boric acid (99\%), acetic acid $(99.8 \%)$ and borax (98\%) were acquired from Dynamic (São Paulo, Brazil), methanol and acetonitrile of HPLC grade were obtained from J. T. Baker (Phillipsburg, USA).

Initially, $10.00 \mathrm{~mL}$ of $1 \mathrm{mg} \mathrm{mL}^{-1}$ creatinine solution was prepared (stock solution) and an aliquot of this was used to prepare a $2000 \mathrm{ng} \mathrm{mL}^{-1}$ solution, which was used in the optimization step. The acetate buffer solution $(\mathrm{pH} 3)$ and borate solution $(\mathrm{pH} 9)$ were prepared using the appropriate amounts of acid and salt to obtain $1 \mathrm{~L}$ at a concentration of $0.25 \mathrm{~mol} \mathrm{~L}^{-1}$. The water used to prepare the solutions was first purified by a Milli-Q system (Millipore, São Paulo, Brazil). The tips of DPX containing the extraction RP (styrene divinylbenzene) and C-18 phases were provided by Gerstel ${ }^{\circledR}$ (Linthicum, MD, United States).

\section{Optimization of the DPX process}

In order to evaluate the sorption equilibrium of the DPX extraction process and perform its time analysis, a $2^{4}$ full factorial design was carried out using the following parameters: $\mathrm{pH}$ of the samples ( 3 and 9), type of desorption solvent (acetonitrile and methanol), and the number of draw/ eject cycles in both adsorption and desorption (1 and 7), using $10 \mathrm{mg}$ of sorbent in the DPX tips and duration of $30 \mathrm{~s}$ for each extraction/desorption. All experiments were performed randomly, including replicates. Table 1 presents the factorial design runs. Calculations were run in Design-Expert software, version 9. ${ }^{24}$ The efficiency in Table 1 was estimated by obtained peak area values of the obtained chromatograms from creatinine standard solution in concentration of $500 \mathrm{ng} \mathrm{L}^{-1}$ after the DPX process. The highest area value was considered $100 \%$ and following values were estimated related to this.

Instrument and chromatographic conditions

The LC-UV analyses were performed on a 1220 Infinity LC instrument (Agilent Technologies) equipped with a UV-Vis detector with variable wavelength $(\lambda=260 \mathrm{~nm})$. The separation was performed in an Agilent ZORBAX Eclipse XDB-C18 $(4.6 \times 150 \mathrm{~mm}, 5 \mu \mathrm{m})$ analytical column at room temperature $\left(25^{\circ} \mathrm{C}\right)$; the mobile phase consisted of an acid solution ( $\mathrm{pH} 4.0$, trifluoroacetic acid) and acetonitrile $(70: 30, \mathrm{v} / \mathrm{v})$ in gradient mode, with a flow-rate 
Table 1. Experimental conditions used in planning and their efficiency

\begin{tabular}{lccccc}
\hline $\mathrm{pH}$ & Draw/eject adsorption cycle & Desorption solvent & Draw/eject adsorption cycle & Peak area $\times 10^{6}$ & Efficiency / \% \\
\hline 3 & 1 & methanol & 1 & 8.6011 & 80.47 \\
9 & 1 & methanol & 1 & 9.7533 & 91.25 \\
3 & 7 & methanol & 1 & 8.5014 & 79.53 \\
9 & methanol & 1 & 9.7987 & 91.67 \\
3 & 7 & acetonitrile & 1 & 7.1712 & 67.01 \\
9 & 1 & acetonitrile & 1 & 8.5124 & 79.64 \\
3 & 1 & acetonitrile & 1 & 7.8974 & 73.88 \\
9 & 7 & acetonitrile & 1 & 8.1457 & 76.21 \\
3 & methanol & 7 & 9.8261 & 91.93 \\
9 & 7 & methanol & 7 & 10.689 & 100.00 \\
3 & 1 & methanol & 7 & 8.9754 & 83.97 \\
9 & 1 & methanol & 7 & 9.1435 & 85.54 \\
3 & 7 & acetonitrile & 7 & 8.4396 & 78.96 \\
9 & 7 & acetonitrile & 7 & 5.0831 & 47.55 \\
3 & 1 & acetonitrile & 7 & 6.9876 & 65.37 \\
9 & 7 & acetonitrile & 7 & 7.8974 & 73.88 \\
\hline
\end{tabular}

of $1.0 \mathrm{~mL} \mathrm{~min} \mathrm{~m}^{-1}$ for $2 \mathrm{~min}$, after which the flow-rate was reduced to $0.5 \mathrm{~mL} \mathrm{~min}^{-1}$ up to $5 \mathrm{~min}$.

\section{Synthetic urine}

For the optimization and analytical validation assays, synthetic urine was used. The synthetic urine was prepared by dissolving $3.333 \mathrm{~g} \mathrm{~L}^{-1}$ of urea $\left(\mathrm{CH}_{4} \mathrm{~N}_{2} \mathrm{O}\right), 0.050 \mathrm{~g} \mathrm{~L}^{-1}$ of uric acid $\left(\mathrm{C}_{5} \mathrm{H}_{4} \mathrm{~N}_{4} \mathrm{O}_{3}\right), 0.177 \mathrm{~g} \mathrm{~L}^{-1}$ of creatinine $\left(\mathrm{C}_{4} \mathrm{H}_{7} \mathrm{~N}_{3} \mathrm{O}\right)$, $1.000 \mathrm{~g} \mathrm{~L}^{-1}$ of chloride $\left(\mathrm{Cl}^{-}\right), 1.000 \mathrm{~g} \mathrm{~L}^{-1}$ of potassium $\left(\mathrm{K}^{+}\right)$, $0.025 \mathrm{~g} \mathrm{~L}^{-1}$ of phosphate $\left(\mathrm{PO}_{4}{ }^{3-}\right), 0.300 \mathrm{~g} \mathrm{~L}^{-1}$ of sulfate $\left(\mathrm{SO}_{4}^{2-}\right), 0.025 \mathrm{~g} \mathrm{~L}^{-1}$ of calcium $\left(\mathrm{Ca}^{2+}\right), 0.167 \mathrm{~g} \mathrm{~L}^{-1}$ of magnesium $\left(\mathrm{Mg}^{2+}\right), 0.167 \mathrm{~g} \mathrm{~L}^{-1}$ of sodium $\left(\mathrm{Na}^{+}\right), 0.025 \mathrm{~g} \mathrm{~L}^{-1}$ of ammonium $\left(\mathrm{NH}_{4}^{+}\right)$, and $0.167 \mathrm{~g} \mathrm{~L}^{-1}$ of carbonates $\left(\mathrm{CO}_{3}^{2-}\right)$ in $1 \mathrm{~L}$ of ultra-purified water. ${ }^{25}$ These salts were obtained from Dynamic (São Paulo, Brazil).

\section{Urine samples}

Urine samples from patients who had collected urine for employee health tests and who had not been exposed to any drug for at least $72 \mathrm{~h}$ were kindly supplied by Secretaria de Saúde Municipal de Jataí, Goiás State, Brazil. These solutions were stable for four days at $2-8{ }^{\circ} \mathrm{C}$.

\section{Analytical validation}

Analytical curves were constructed using linear regression of the creatinine peak areas (Y) against creatinine urine concentration $\left(\mathrm{X}, \mathrm{g} \mathrm{L}^{-1}\right)$, to evaluate the linearity. The accuracy and inter-day precision were determined by means of quintuplicate assays of the blank synthetic urine samples spiked with creatinine solution, representing the entire range of the creatinine biomonitoring concentration in urine for men and women. Accuracy values were calculated by comparing the concentrations of creatinine solution added to the urine samples with spiked urine concentrations determined by the analytical curve.

\section{Results and Discussion}

\section{Optimization of the DPX conditions}

Prior to the optimization step, two DPX extraction phases were evaluated to creatinine analyses. To evaluate the DPX phase performance, C-18 and RP (styrene divinylbenzene) phases were submitted to extraction under the same conditions: $\mathrm{pH} 7$, methanol for solvent desorption, and three draw/eject adsorption and desorption cycles. According to the obtained results, the RP phase showed better extraction efficiency under this condition, based on peak area results. The retention of the analyte is considered as the result of three contributions: filling of the fixed pores, expansion of the fixed and collapsed pores and swelling of the polymer array, as well as polymer and analyte chemical structure. ${ }^{26,27}$ The RP phase used is composed essentially of styrene divinylbenzene copolymer, more hydrophobic than $\mathrm{C} 18$ phases, composed by octadecilsilane and residual 
silane groups. ${ }^{28}$ Considering the structure and electron distribution into styrene divinylbenzene polymer net and creatinine molecules showed in Figure 1, probably the $\pi-\pi$ interaction contributed to higher extraction efficiency of RP phase, and it was selected to conduct the subsequent extractions. (a)

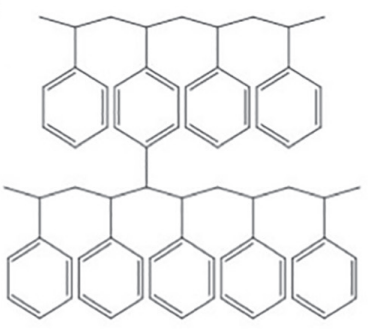

(b)

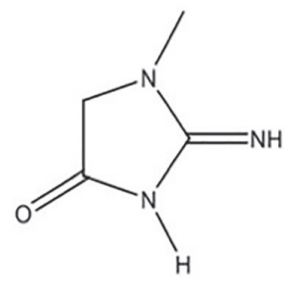

Figure 1. Representation of the structure of: (a) styrene/divinylbenzene polymer and (b) creatinine molecule.

The sorption capacity, method sensitivity, and carryover are important parameters that should be considered during the development of new methods. Using the selected phase, RP, the DPX conditions were evaluated based on the extraction efficiency using experimental design (Table 1). The levels of normal creatinine concentration were considered during the optimization of the DPX parameters.

Pareto analysis of the results presented in Table 1 and Figure 2 reveals that the main factor affecting DPX extraction is the type of desorption solvent (C) and that it has a synergic significant effect when combined with the number of desorption cycles $(\mathrm{CD})$ and with the $\mathrm{pH}$ of the samples (ACD). The adsorption cycle (B) did not present any significant main or interaction/synergic effect.

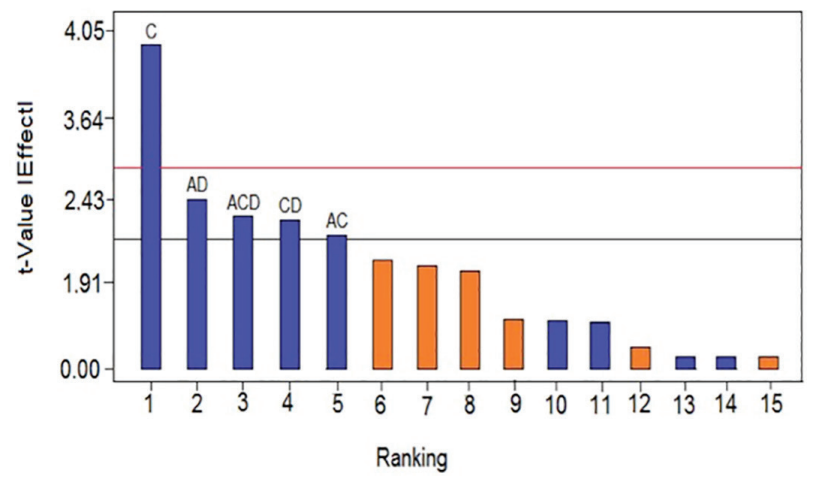

Figure 2. Pareto chart for the factorial design of Table 1. The levels A, $\mathrm{B}, \mathrm{C}$, and $\mathrm{D}$ are the factors shown in Table 2.

Table 2. Factors and levels with corresponding variables

\begin{tabular}{lc}
\hline Level & Factor \\
\hline A & pH \\
B & draw/eject adsorption cycles \\
C & desorption solvent \\
D & draw/eject desorption cycles \\
\hline
\end{tabular}

According to the software Desing-Expert ${ }^{\circledR 24}$ results a higher extraction efficiency was obtained with $500 \mu \mathrm{L}$ of sample diluted with borate solution at $\mathrm{pH} 9$ with a single draw/eject extraction cycle. After pre-concentration, the analytes were desorbed in $1 \mathrm{~mL}$ of methanol in seven draw/eject cycles.

At $\mathrm{pH}$ values above $\mathrm{p} K_{\mathrm{a}}$ molecule it is possible to elute the neutral or partially ionized creatinine molecules. Once the extraction was performed in solution $\mathrm{pH} 9$, creatinine molecules was essentially neutral form $\left(\mathrm{p} K_{\mathrm{a}} 5.02\right)$, which contributed to higher extraction efficiency since the DPX used phase was based on styrene divynylbenzene groups. ${ }^{29}$

\section{Analytical validation}

The selectivity of the developed method is shown by the representative chromatograms in Figure 3, illustrating synthetic urine without creatinine and synthetic urine spiked with $0.317 \mathrm{~g} \mathrm{~L}^{-1}$ of creatinine. These chromatograms do not present interfering peaks at the retention time of the creatinine. The synthetic urine consisted of a solution with ionic strength and viscosity similar to those of human urine, but without the presence of human creatinine.

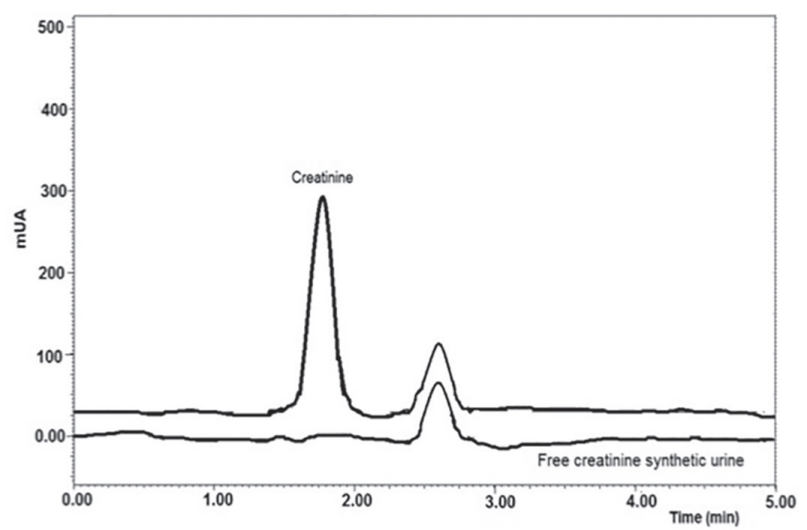

Figure 3. DPX/LC-UV chromatograms of free creatinine synthetic urine and synthetic urine enriched with $0.317 \mathrm{~g} \mathrm{~L}^{-1}$ of creatinine, $\lambda=260 \mathrm{~nm}$.

The analytical signals relating to the same interference as the possible analyte retention time were less than $20 \%$ of the chromatographic sign creatinine concentration, corresponding to the concentration at the limit of quantification (LOQ).

The linearity of the DPX/LC-UV method ranged from the LOQ $\left(0.317 \mathrm{~g} \mathrm{~L}^{-1}\right)$ to $3.390 \mathrm{~g} \mathrm{~L}^{-1}$ of creatinine. The analysis of variance (ANOVA) for the calibration curve points resulted in the regression equation $\mathrm{y}=2.586 \times 10^{6}+3.061 \times 10^{7} \mathrm{x}$ with $\mathrm{r}^{2}=0.9962$ and a $p$-value of $6 \times 10^{-5}$, showing that the linear model is highly significant. Each point of the calibration curve was performed in five replicates. 
The LOQ was determined as the lowest concentration on the calibration curve, for which the coefficient of variation $(\mathrm{CV})$ was less than $8.8 \%$ (Table 3 ), on the basis of a signal-to-noise ratio of approximately 10 . According to this, the LOQ obtained under the experimental condition was $0.317 \mathrm{~g} \mathrm{~L}^{-1}$.

Table 3. Inter-day precision (coefficient of variation) and accuracy of the DPX/LC-UV method for creatinine

\begin{tabular}{lccc}
\hline $\begin{array}{l}\text { Concentration / } \\
\left(\mathrm{g} \mathrm{L}^{-1}\right)\end{array}$ & $\mathrm{CV} / \%$ & Accuracy /\% & $\begin{array}{c}\text { Relative } \\
\text { recovery /\% }\end{array}$ \\
\hline 0.34 & 8.8 & 90.6 & 93.6 \\
1.90 & 3.9 & 88.3 & 87.6 \\
3.40 & 3.3 & 85.5 & 88.0 \\
\hline
\end{tabular}

$\mathrm{CV}$ : coefficient of variation.

The accuracy and inter-day precision of the DPX/LC-UV method were assessed via replicate analysis $(n=5)$ using synthetic urine samples spiked with creatinine standard solutions at various concentrations (Table 3). The precision was determined according to the $\mathrm{CV}$ percentage (inter-day) at three levels. The $\mathrm{CV}$ percentage values ranged from 3.3 to $8.8 \%$ for creatinine (Table 3 ). The relative recovery of the developed method was assessed via replicate analysis $(n=5)$ of the urine samples spiked with standards at three different concentrations (Table 3). The recovery values obtained ranged from 87.6 to $93.6 \%$. As shown in Table 3 , the relative recoveries for creatinine were quite consistent between matrices and spiking levels, and as expected, relative standard deviations (RSDs) decreased as the spiking concentrations increased.

Song and co-workers ${ }^{30}$ developed a method for creatinine analysis using liquid chromatography-mass spectrometry (LC-MS) with mobile phase methanol:acetonitrile $55: 45(\mathrm{v} / \mathrm{v})$ and a flow-rate of $0.3 \mathrm{~mL} \mathrm{~min}^{-1}$ to result in a retention time of approximately 1.5 and $3.0 \mathrm{~min}$. Even using an higher flow-rate, which could make the developed method less attractive, the mobile phase consisted of ultrapure water acidified to $\mathrm{pH} 4$ and acetonitrile $(70: 30 \mathrm{v} / \mathrm{v})$ with a spectrophotometric detector, which has a lower cost comparing to MS detector. Thus the DPX/LC-UV developed method was considered less expensive and also efficient, allowing quick determination of creatinine in urine samples.

To evaluate the proposed method for clinical use, the described protocol was applied to the analysis of urine samples from patients participating in employee health checks who had not been exposed to any drug for at least $72 \mathrm{~h}$. The urine samples were collected from patients who had been exposed to high levels of hexane vapor. The literature appoints increased creatinine levels in these patients, so this samples were diluted ten times before being extracted by DPX, and was possible to determine their concentration within the analytical curve. Twenty-four samples were analyzed and the creatinine concentrations found in these samples by correlation values ranged from 3.9 to $18.2 \mathrm{~g} \mathrm{~L}^{-1}$. Figure 4 shows some chromatograms obtained from the patients' urine analysis. The peak shapes and resolution of the obtained chromatograms are very similar to those obtained using spiked urine samples, and no interference was observed.

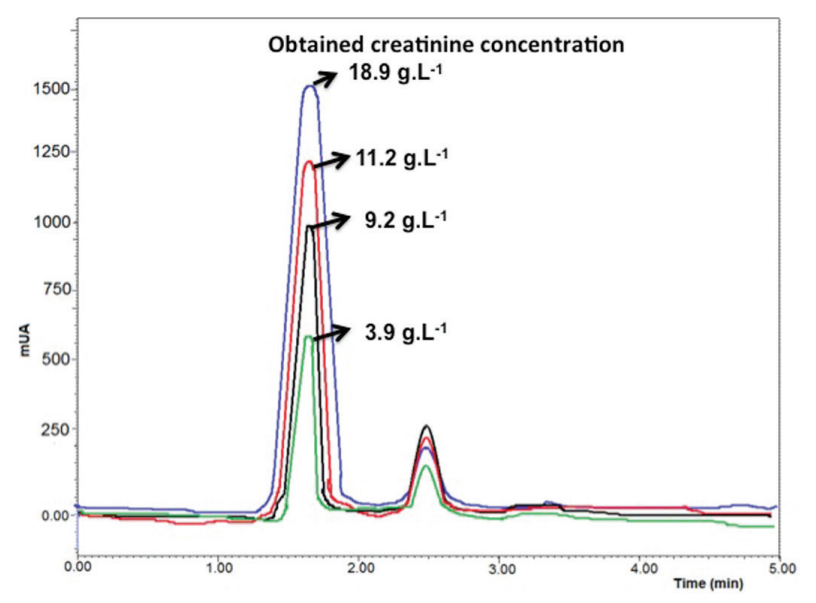

Figure 4. DPX/LC-UV $(\lambda=260 \mathrm{~nm})$ analysis of urine sample from patients who had not been exposed to any drug for at least $72 \mathrm{~h}$.

The entire DPX process presented here, including the conditioning, sample loading, elution, and washing steps, requires approximately $3 \mathrm{~min}$. This is a significant reduction in time compared to the traditional SPE method (approximately $20 \mathrm{~min}){ }^{31}$

\section{Conclusions}

The DPX/LC-UV method developed here offers a good alternative that could replace the colorimetric method used in clinical analysis, since, in addition to the low solvent consumption and shorter analysis time, by using the experimental design it was possible to determine the optimum extraction conditions with fewer experiments than would be used in a one-dimensional optimization.

Based on the analytical validation results, the proposed method can be a useful tool for determining the creatinine levels in urine samples. The developed DPX/LC-UV method was used for creatinine determination in urine samples of patients participating in employee health checks who showed high creatinine concentrations in their urine samples, which could be attributed, among other things, to the higher exposure of these patients to hexane vapors. 
More clinical assays should be performed to confirm this association.

\section{Acknowledgments}

This work was supported by grants from Conselho Nacional de Desenvolvimento Científico e Tecnológico (CNPq). The authors thank the Grupo de Métodos Eletroforéticos (GME) of Universidade Federal de Goiás for donating the creatinine standard and the Secretaria Municipal de Saúde de Jataí (Goiás State) and Prof Dr Cleber Ramos (in memoriam) for donating the urine samples.

\section{References}

1. Panasyuk-Delaney, T.; Mirsky, V. M.; Wolfbeis, O. S.; Electroanalysis 2002, 14, 221.

2. Randviir, E. P.; Banks, C. E.; Sens. Actuators, B 2013, 183, 239.

3. Koncki, R.; Trends Anal. Chem. 2008, 27, 304.

4. de Araújo, W. R.; Salles, M. O.; Paixão, T. R. L. C.; Sens. Actuators, B 2012, 173, 847.

5. Bhagavan, N. V.; Medical Biochemistry, $4^{\text {th }}$ ed.; Harcourt Academic Press: Orlando, FL, USA, 2002.

6. Myers, G. L.; Miller, W. G.; Coresh, J. J.; Fleming, N.; Greenberg, T.; Clin. Chem. 2006, 52, 5.

7. Tsuchida, T.; Yoda, K.; Clin. Chem. 1983, 29, 51.

8. Madaras, M. B.; Popescu, I. C.; Ufer, S.; Buck, R. P.; Anal. Chim. Acta 1996, 319, 335.

9. Khan, G. F.; Wernet, W.; Anal. Chim. Acta 1997, 351, 151.

10. Osaka, T.; Komaba, S.; Amano, A.; Electrochem. Soc. 1998, $145,406$.

11. Killard, A. J.; Smyth, M. R.; Trends Biotechnol. 2000, 18, 433.

12. Lad, U.; Khokhar, S.; Kale, G. M.; Anal. Chem. 2008, 80, 7910.

13. Mohabbati-Kalejahi, E.; Azimirad, V.; Bahrami, M.; Ganbari, A.; Talanta 2012, 97, 1.

14. Smith-Palmer, T.; J. Chromatogr. B 2002, 781, 93.
15. Teichert, F.; Verschoyle1, R. D.; Greaves, P.; Thorpe, J. F.; Mellon, J. K.; Steward, W. P.; Farmer, P. B.; Gescher, A. J.; Singh, R.; Rapid Commun. Mass Spectrom. 2009, 23, 258.

16. Jiang, Y.; Cheng, X.; Wang, C.; Ma, Y.; Anal. Chem. 2010, 82, 9022.

17. Resines, J. A.; Arin, M. J.; Diez, M. T.; Del Moral, P. G.; J. Liq. Chromatogr. Relat. Technol. 1999, 22, 2503.

18. Pena-Abaurrea, M.; de la Torre, V. S. G.; Ramos, L.; J. Chromatogr. A 2013, 1313, 103.

19. Ellison, S. T.; Brewer, W. E.; Morgan, S. L.; J. Anal. Toxicol. 2009, 33, 356.

20. Kumazawa, T.; Hasegawa, C.; Lee, X.-P.; Hara, K.; Seno, H.; Suzuki, O.; Sato, K.; J. Pharm. Biomed. Anal. 2007, 44, 602.

21. Keough, T.; Lacey, M. P.; Youngquist, R. S.; Rapid Commun. Mass Spectrom. 2002, 16, 1003.

22. Lehotay, S. J.; Mastovska, K.; Lightfield, A. R.; Nunez, A.; Dutko, T.; Ng, C.; Bluhm, L.; J. Chromatogr. A 2013, 1313, 103.

23. Mozaner Bordin, D. C.; Alves, M. N. R.; Cabrices, O. G.; de Campos, E. G.; de Martinis, B. S.; J. Anal. Toxicol. 2014, 38, 31.

24. Design-Expert, version 9, Stat-Ease, Minneapolis, MN, USA, 2012.

25. Dbira, S.; Cañizares, P.; Rodrigo, M. A.; J. Electroanal. Chem. 2015, 744, 62 .

26. Coutinho, F. M. B.; Neves, M. A. F. S.; Dias, M. L.; J. Appl. Polym. Sci. 1998, 67, 781.

27. Pinto, M.; Queiroz, M. E. C.; Sci. Chromatogr. 2015, 7, 101.

28. Smet, S. D.; Lyen, F.; J. Chromatogr. A 2015, 1404, 51.

29. Ambrose, R. T.; Ketchum, D. F.; Smith, J. W.; Clin. Chem. 1983, 29, 2.

30. Ou, M.; Song, Y.; Li, S.; Liu, G.; Jia, J.; Zhang, M.; Zhang, H.; Yu, C.; PLoS One 2015, 133912, 1.

31. Huang, Z.; Zhang, S.; J. Chromatogr. B 2003, 792, 241.

Submitted: July 18, 2017 Published online: October 17, 2017 